February, 1996

DESY 96-031

HLRZ 96-11

HUB-EP-96/4

\title{
Lattice Operators for Moments of the Structure Functions and their Transformation under the Hypercubic Group
}

\author{
M. Göckeler ${ }^{1,2}$, R. Horsley ${ }^{3}$, E.-M. Ilgenfritz ${ }^{3}$, H. Perlt ${ }^{4}$, P. Rakow ${ }^{1}$, \\ G. Schierholz ${ }^{5,1}$ and A. Schiller ${ }^{4}$ \\ ${ }^{1}$ HLRZ, c/o Forschungszentrum Jülich, D-52425 Jülich, Germany \\ ${ }^{2}$ Institut für Theoretische Physik, RWTH Aachen, \\ D-52056 Aachen, Germany \\ ${ }^{3}$ Institut für Physik, Humboldt-Universität, D-10115 Berlin, Germany \\ ${ }^{4}$ Institut für Theoretische Physik, Universität Leipzig, \\ D-04109 Leipzig, Germany \\ ${ }^{5}$ Deutsches Elektronen-Synchrotron DESY, D-22603 Hamburg, Germany
}

\begin{abstract}
For lattice operators that are relevant to the calculation of moments of nucleon structure functions we investigate the transformation properties under the hypercubic group. We give explicit bases of irreducible subspaces for tensors of rank $\leq 4$.
\end{abstract}




\section{Introduction}

The calculation of the moments of hadronic structure functions by Monte Carlo simulations of lattice QCD requires the study of matrix elements of composite operators appearing in the operator product expansion of the appropriate currents [1, 2, 3, 4]. In the continuum, these operators are classified according to their behaviour under Lorentz transformations and charge conjugation. In particular, the leading twist $(=2)$ operators are totally symmetric and traceless Lorentz tensors. Due to their transformation properties they cannot mix with lower-dimensional operators under renormalization.

When QCD is put on the lattice, the necessary analytic continuation from Minkowski to Euclidean space replaces the Lorentz group by the orthogonal group $O(4)$, which by the discretization of space-time is further reduced to the hypercubic group $H(4) \subset O(4)$. Hence the lattice operators have to be classified according to $H(4)$ and one should only work with operators which transform irreducibly under $H(4)$. Since $H(4)$ is only a finite group, the restrictions imposed by symmetry are less stringent than in the continuum and the possibilities for mixing increase. A control over the mixing problem is however vital for a successful lattice calculation. In particular, mixing with lower-dimensional operators presents special difficulties and is to be avoided whenever possible. Therefore a computation of structure functions on the lattice requires a study of the $H(4)$-transformation properties of the relevant lattice operators.

In the present paper we give explicit bases for $H(4)$-irreducible subspaces of operators up to spin 4 . We consider the following types of operators:

$$
\begin{gathered}
\mathcal{O}_{\mu_{1} \mu_{2} \ldots \mu_{n}}^{0}:=\bar{\psi} \gamma_{\mu_{1}} \overleftrightarrow{D}_{\mu_{2}} \overleftrightarrow{D}_{\mu_{3}} \ldots \overleftrightarrow{D}_{\mu_{n}} \psi \\
\mathcal{O}_{\mu_{1} \mu_{2} \ldots \mu_{n}}^{5}:=\bar{\psi} \gamma_{\mu_{1}} \gamma_{5} \overleftrightarrow{D}_{\mu_{2}} \overleftrightarrow{D}_{\mu_{3}} \ldots \overleftrightarrow{D}_{\mu_{n}} \psi \\
\mathcal{O}_{\mu_{1} \mu_{2} \ldots \mu_{n}}^{g}:=\operatorname{tr} \sum_{\alpha=1}^{4} G_{\mu_{1} \alpha} D_{\mu_{2}} \cdots D_{\mu_{n-1}} G_{\mu_{n} \alpha}
\end{gathered}
$$

Here $\psi$ is a Wilson fermion field, $D_{\mu}$ denotes the lattice covariant derivative and $\overleftrightarrow{D}_{\mu}=\vec{D}_{\mu}-\overleftarrow{D}_{\mu}$ (for the proper lattice definition of expressions like $\vec{D}_{\mu} \overleftarrow{D}_{\nu}$ see [3] $)$ 
The (clover) lattice version $G_{\mu \nu}$ of the gluon field strength is given by

$$
\begin{array}{rl}
G_{\mu \nu}(x)=\frac{\mathrm{i}}{8} \sum_{s, s^{\prime}= \pm 1} & s s^{\prime} U(x, x+s \hat{\nu}) U\left(x+s \hat{\nu}, x+s \hat{\nu}+s^{\prime} \hat{\mu}\right) \\
& \times U\left(x+s \hat{\nu}+s^{\prime} \hat{\mu}, x+s^{\prime} \hat{\mu}\right) U\left(x+s^{\prime} \hat{\mu}, x\right)+\text { h.c. }
\end{array}
$$

where we have defined the parallel transporter $U(x, y)$ for nearest-neighbour lattice points $x, y$ as

$$
U(x, y)= \begin{cases}U(x, \mu) & \text { if } y=x+\hat{\mu} \\ U(x-\hat{\mu}, \mu)^{+} & \text {if } y=x-\hat{\mu} .\end{cases}
$$

\section{Transformation Properties}

Charge conjugation operates on the fermion fields $\psi(x), \bar{\psi}(x)$ and on the lattice gauge field $U(x, \mu)$ according to

$$
\begin{aligned}
\psi(x) & \rightarrow C \bar{\psi}(x)^{T}, \\
\bar{\psi}(x) & \rightarrow-\psi(x)^{T} C^{-1}, \\
U(x, \mu) & \rightarrow U(x, \mu)^{*}
\end{aligned}
$$

with the charge conjugation matrix $C$ satisfying

$$
C \gamma_{\mu}^{T} C^{-1}=-\gamma_{\mu}
$$

and we get

$$
\begin{gathered}
\mathcal{O}_{\mu_{1} \mu_{2} \ldots \mu_{n}}^{0} \rightarrow(-1)^{n} \mathcal{O}_{\mu_{1} \mu_{n} \mu_{n-1} \ldots \mu_{2}}^{0}, \\
\mathcal{O}_{\mu_{1} \mu_{2} \ldots \mu_{n}}^{5} \rightarrow(-1)^{n-1} \mathcal{O}_{\mu_{1} \mu_{n} \mu_{n-1} \ldots \mu_{2}}^{5}, \\
\mathcal{O}_{\mu_{1} \mu_{2} \ldots \mu_{n}}^{g} \rightarrow \mathcal{O}_{\mu_{1} \mu_{2} \ldots \mu_{n}}^{g} .
\end{gathered}
$$

Note that replacing $\overleftrightarrow{D}_{\mu}$ by $\vec{D}_{\mu}$ or $\overleftarrow{D}_{\mu}$ in (11.1), (1.2) leads to operators that have no simple behaviour under charge conjugation.

In the study of the transformation properties under $H(4)$ we use the notation of ref. [5] and consider

$$
H(4)=\left\{(a, \pi) \mid a \in Z_{2}^{4}, \pi \in S_{4}\right\}
$$


So $\pi$ is a permutation of four elements and $a_{\mu} \in\{0,1\}$. For elements of the symmetric group $S_{n}$ we shall use the cycle notation (see, e.g., [6]). In terms of an orthonormal basis $v_{1}, \ldots, v_{4}$ of a four-dimensional vector space the defining representation $T$ of $H(4)$ is given by:

$$
T(a, \pi) v_{\mu}=(-1)^{a_{\mu}} v_{\pi(\mu)} .
$$

The corresponding orthogonal $4 \times 4$ matrices are

$$
T(a, \pi)_{\mu \nu}=(-1)^{a_{\nu}} \delta_{\mu, \pi(\nu)}
$$

Note that $H(4)$ is generated by the three elements

$$
\begin{aligned}
& \alpha=((0,0,0,0),(12)), \\
& \beta=((0,0,0,0),(2341)), \\
& \gamma=((1,0,0,0), \mathrm{id}) .
\end{aligned}
$$

The corresponding matrices are given by

$$
\begin{aligned}
& T(\alpha)=\left(\begin{array}{llll}
0 & 1 & 0 & 0 \\
1 & 0 & 0 & 0 \\
0 & 0 & 1 & 0 \\
0 & 0 & 0 & 1
\end{array}\right), \\
& T(\beta)=\left(\begin{array}{llll}
0 & 0 & 0 & 1 \\
1 & 0 & 0 & 0 \\
0 & 1 & 0 & 0 \\
0 & 0 & 1 & 0
\end{array}\right), \\
& T(\gamma)=\left(\begin{array}{llll}
-1 & 0 & 0 & 0 \\
0 & 1 & 0 & 0 \\
0 & 0 & 1 & 0 \\
0 & 0 & 0 & 1
\end{array}\right) .
\end{aligned}
$$

On our operators the element $(a, \pi) \in H(4)$ acts as follows:

$$
\mathcal{O}_{\mu_{1} \mu_{2} \ldots \mu_{n}}^{0} \rightarrow(-1)^{a_{\mu_{1}}+a_{\mu_{2}}+\cdots+a_{\mu_{n}}} \mathcal{O}_{\pi\left(\mu_{1}\right) \pi\left(\mu_{2}\right) \ldots \pi\left(\mu_{n}\right)}^{0},
$$




$$
\begin{gathered}
\mathcal{O}_{\mu_{1} \mu_{2} \ldots \mu_{n}}^{g} \rightarrow(-1)^{a_{\mu_{1}}+a_{\mu_{2}}+\cdots+a_{\mu_{n}}} \mathcal{O}_{\pi\left(\mu_{1}\right) \pi\left(\mu_{2}\right) \ldots \pi\left(\mu_{n}\right)}^{g}, \\
\mathcal{O}_{\mu_{1} \mu_{2} \ldots \mu_{n}}^{5} \rightarrow(-1)^{a_{\mu_{1}}+a_{\mu_{2}}+\cdots+a_{\mu_{n}}} \operatorname{det}(T(a, \pi)) \mathcal{O}_{\pi\left(\mu_{1}\right) \pi\left(\mu_{2}\right) \ldots \pi\left(\mu_{n}\right)}^{5} .
\end{gathered}
$$

So both $\mathcal{O}^{0}$ and $\mathcal{O}^{g}$ transform according to the $n$-th tensor power of $T$. The operators $\mathcal{O}^{5}$, on the other hand, have one factor of $T$ replaced by $T \cdot \operatorname{det} T$. Therefore these tensor products of $H(4)$ representations have to be decomposed into irreducible components. In doing so we consider the operators (1.1) ((1.2), respectively) as forming an orthonormal basis of the representation space. The bases for $H(4)$ irreducible subspaces to be given below will also be chosen orthonormal. We shall present our results only for $\mathcal{O}^{0}$, the formulas for $\mathcal{O}^{g}$ are identical, except for the $C$-parity.

\section{Irreducible Representations and Decomposition}

The 20 (inequivalent) irreducible representations of $H(4)$ are denoted by $\tau_{k}^{(l)}$, where $l$ is the dimension of the representation and $k=1,2, \ldots$ distinguishes inequivalent representations of the same dimension. There are four one-dimensional representations, two of dimension two, four of dimension three, four and six, and two of dimension eight. The defining representation $T$ will be labeled as $\tau_{1}^{(4)}$ and the representation $T \cdot \operatorname{det} T$ as $\tau_{4}^{(4)}$. For the reader's convenience, we give in Table 1 the correspondence with the notation of ref. [7]. In the following, the symbol "=" used for representations only indicates equivalence and not actual identity of the representations.

For the tensor products of $\tau_{1}^{(4)}$ with itself we have the following decompositions in the cases $n=2,3,4$ :

$$
\begin{aligned}
\tau_{1}^{(4)} \otimes & \tau_{1}^{(4)}=\tau_{1}^{(1)} \oplus \tau_{1}^{(3)} \oplus \tau_{1}^{(6)} \oplus \tau_{3}^{(6)}, \\
\tau_{1}^{(4)} \otimes & \tau_{1}^{(4)} \otimes \tau_{1}^{(4)}=4 \tau_{1}^{(4)} \oplus \tau_{2}^{(4)} \oplus \tau_{4}^{(4)} \oplus 3 \tau_{1}^{(8)} \oplus 2 \tau_{2}^{(8)}, \\
\tau_{1}^{(4)} \otimes & \tau_{1}^{(4)} \otimes \tau_{1}^{(4)} \otimes \tau_{1}^{(4)} \\
= & 4 \tau_{1}^{(1)} \oplus \tau_{2}^{(1)} \oplus \tau_{4}^{(1)} \oplus 3 \tau_{1}^{(2)} \oplus 2 \tau_{2}^{(2)} \oplus 7 \tau_{1}^{(3)} \oplus 3 \tau_{2}^{(3)} \oplus 3 \tau_{3}^{(3)} \oplus 3 \tau_{4}^{(3)} \\
& \oplus 10 \tau_{1}^{(6)} \oplus 6 \tau_{2}^{(6)} \oplus 10 \tau_{3}^{(6)} \oplus 6 \tau_{4}^{(6)} .
\end{aligned}
$$

For $n=2,3$ and the totally symmetric tensors of rank 4 one can construct bases for irreducible subspaces simply by inspection. For the not totally symmetric rank-4 


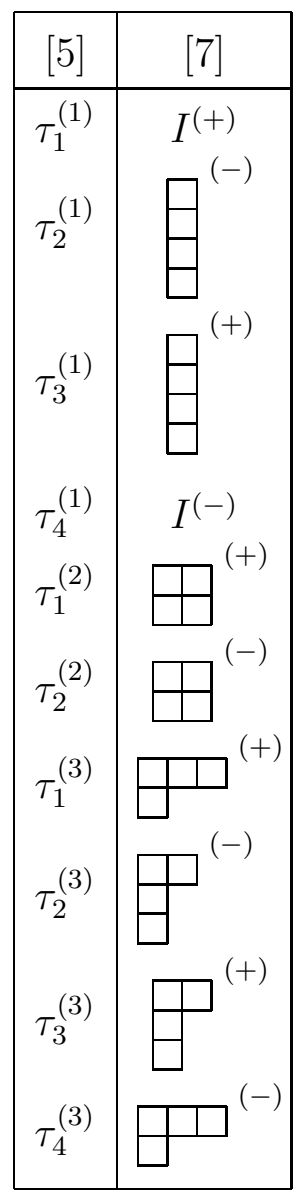

\begin{tabular}{|c|c|}
\hline 5 & 7 \\
\hline$\tau_{1}^{(4)}$ & $\left(\frac{1}{2}, \frac{1}{2}\right)^{(+)}$ \\
\hline$\tau_{2}^{(4)}$ & $\overline{\left(\frac{1}{2}, \frac{1}{2}\right)}^{(-)}$ \\
\hline$\tau_{3}^{(4)}$ & $\overline{\left(\frac{1}{2}, \frac{1}{2}\right)}^{(+)}$ \\
\hline$\tau_{4}^{(4)}$ & $\left(\frac{1}{2}, \frac{1}{2}\right)^{(-)}$ \\
\hline$\tau_{1}^{(6)}$ & $(10) \oplus(01)$ \\
\hline$\tau_{2}^{(6)}$ & $(\overline{10}) \oplus(\overline{01})$ \\
\hline$\tau_{3}^{(6)}$ & $6^{(+)}$ \\
\hline$\tau_{4}^{(6)}$ & $6^{(-)}$ \\
\hline$\tau_{1}^{(8)}$ & $8^{(+)}$ \\
\hline$\tau_{2}^{(8)}$ & $8^{(-)}$ \\
\hline
\end{tabular}

Table 1: Irreducible representations of $H(4)$.

tensors we have to proceed more systematically as will be explained below. In the end one obtains a list of operators which have definite charge conjugation parity $C$ and transform irreducibly under $H(4)$. Furthermore, they can be classified as being traceless (i.e. all possible traces vanish) or having nonvanishing trace. They also belong to definite irreducible representations of the symmetric group $S_{n}$ acting according to

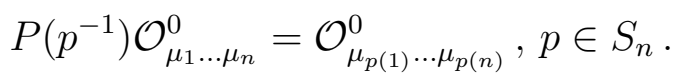

Hence the operators are symmetric or antisymmetric in their indices, or they transform according to some higher-dimensional irreducible representation of $S_{n}$. Symmetrisation will be denoted by $\{\cdots\}$, i.e.

$$
\mathcal{O}_{\left\{\mu_{1} \mu_{2} \ldots \mu_{n}\right\}}=\frac{1}{n !} \sum_{p \in S_{n}} \mathcal{O}_{\mu_{p(1)} \mu_{p(2)} \ldots \mu_{p(n)}}
$$




\section{Irreducible Bases}

One finds the following bases transforming irreducibly under $H(4)$.

\section{1 $n=1$}

$\tau_{1}^{(4)}, C=-1:$

$$
\mathcal{O}_{\mu}^{0}, 1 \leq \mu \leq 4
$$

\section{$4.2 n=2$}

trace $\neq 0$, symmetric, $\tau_{1}^{(1)}, C=+1$ :

$$
\frac{1}{2} \sum_{\mu=1}^{4} \mathcal{O}_{\mu \mu}^{0}
$$

trace $=0$, symmetric, $\tau_{1}^{(3)}, C=+1$ :

$$
\begin{gathered}
\frac{1}{2}\left(\mathcal{O}_{11}^{0}+\mathcal{O}_{22}^{0}-\mathcal{O}_{33}^{0}-\mathcal{O}_{44}^{0}\right) \\
\frac{1}{\sqrt{2}}\left(\mathcal{O}_{33}^{0}-\mathcal{O}_{44}^{0}\right) \\
\frac{1}{\sqrt{2}}\left(\mathcal{O}_{11}^{0}-\mathcal{O}_{22}^{0}\right)
\end{gathered}
$$

trace $=0$, symmetric, $\tau_{3}^{(6)}, C=+1$ :

$$
\frac{1}{\sqrt{2}}\left(\mathcal{O}_{\mu \nu}^{0}+\mathcal{O}_{\nu \mu}^{0}\right), 1 \leq \mu<\nu \leq 4
$$

trace $=0$, antisymmetric, $\tau_{1}^{(6)}, C=+1$ :

$$
\frac{1}{\sqrt{2}}\left(\mathcal{O}_{\mu \nu}^{0}-\mathcal{O}_{\nu \mu}^{0}\right), 1 \leq \mu<\nu \leq 4 .
$$




\section{$4.3 n=3$}

In order to simplify the notation we define for the case $n=3$

$$
\begin{gathered}
\mathcal{O}_{\left|\nu_{1} \nu_{2} \nu_{3}\right|}=\mathcal{O}_{\nu_{1} \nu_{2} \nu_{3}}-\mathcal{O}_{\nu_{1} \nu_{3} \nu_{2}}-\mathcal{O}_{\nu_{3} \nu_{1} \nu_{2}}+\mathcal{O}_{\nu_{3} \nu_{2} \nu_{1}}, \\
\mathcal{O}_{\left\|\nu_{1} \nu_{2} \nu_{3}\right\|}=\mathcal{O}_{\nu_{1} \nu_{2} \nu_{3}}-\mathcal{O}_{\nu_{1} \nu_{3} \nu_{2}}+\mathcal{O}_{\nu_{3} \nu_{1} \nu_{2}}-\mathcal{O}_{\nu_{3} \nu_{2} \nu_{1}}-2 \mathcal{O}_{\nu_{2} \nu_{3} \nu_{1}}+2 \mathcal{O}_{\nu_{2} \nu_{1} \nu_{3}}, \\
\mathcal{O}_{\left\langle\nu_{1} \nu_{2} \nu_{3}\right\rangle}=\mathcal{O}_{\nu_{1} \nu_{2} \nu_{3}}+\mathcal{O}_{\nu_{1} \nu_{3} \nu_{2}}+\mathcal{O}_{\nu_{3} \nu_{1} \nu_{2}}+\mathcal{O}_{\nu_{3} \nu_{2} \nu_{1}}-2 \mathcal{O}_{\nu_{2} \nu_{3} \nu_{1}}-2 \mathcal{O}_{\nu_{2} \nu_{1} \nu_{3}}, \\
\mathcal{O}_{\left\langle\left\langle\nu_{1} \nu_{2} \nu_{3}\right\rangle\right\rangle}=\mathcal{O}_{\nu_{1} \nu_{2} \nu_{3}}+\mathcal{O}_{\nu_{1} \nu_{3} \nu_{2}}-\mathcal{O}_{\nu_{3} \nu_{1} \nu_{2}}-\mathcal{O}_{\nu_{3} \nu_{2} \nu_{1}}
\end{gathered}
$$

and get the following bases transforming irreducibly under $H(4)$.

trace $\neq 0$, symmetric, $\tau_{1}^{(4)}, C=-1$ :

$$
\frac{1}{\sqrt{2}} \sum_{\nu=1}^{4} \mathcal{O}_{\{\mu \nu \nu\}}^{0}, 1 \leq \mu \leq 4,
$$

trace $=0$, symmetric, $\tau_{1}^{(4)}, C=-1$ :

$$
\frac{1}{\sqrt{2}}\left(\mathcal{O}_{\{\mu \mu \mu\}}^{0}-\sum_{\substack{\nu=1 \\ \nu \neq \mu}}^{4} \mathcal{O}_{\{\mu \nu \nu\}}^{0}\right), 1 \leq \mu \leq 4,
$$

trace $=0$, symmetric, $\tau_{1}^{(8)}, C=-1$ :

$$
\begin{array}{ll}
\frac{\sqrt{3}}{\sqrt{2}}\left(\mathcal{O}_{\{122\}}^{0}-\mathcal{O}_{\{133\}}^{0}\right), & \frac{1}{\sqrt{2}}\left(\mathcal{O}_{\{122\}}^{0}+\mathcal{O}_{\{133\}}^{0}-2 \mathcal{O}_{\{144\}}^{0}\right), \\
\frac{\sqrt{3}}{\sqrt{2}}\left(\mathcal{O}_{\{211\}}^{0}-\mathcal{O}_{\{233\}}^{0}\right), & \frac{1}{\sqrt{2}}\left(\mathcal{O}_{\{211\}}^{0}+\mathcal{O}_{\{233\}}^{0}-2 \mathcal{O}_{\{244\}}^{0}\right), \\
\frac{\sqrt{3}}{\sqrt{2}}\left(\mathcal{O}_{\{311\}}^{0}-\mathcal{O}_{\{322\}}^{0}\right), & \frac{1}{\sqrt{2}}\left(\mathcal{O}_{\{311\}}^{0}+\mathcal{O}_{\{322\}}^{0}-2 \mathcal{O}_{\{344\}}^{0}\right), \\
\frac{\sqrt{3}}{\sqrt{2}}\left(\mathcal{O}_{\{411\}}^{0}-\mathcal{O}_{\{422\}}^{0}\right), & \frac{1}{\sqrt{2}}\left(\mathcal{O}_{\{411\}}^{0}+\mathcal{O}_{\{422\}}^{0}-2 \mathcal{O}_{\{433\}}^{0}\right),
\end{array}
$$

trace $=0$, symmetric, $\tau_{2}^{(4)}, C=-1$ :

$$
\sqrt{6} \mathcal{O}_{\{234\}}^{0}, \sqrt{6} \mathcal{O}_{\{134\}}^{0}, \sqrt{6} \mathcal{O}_{\{124\}}^{0}, \sqrt{6} \mathcal{O}_{\{123\}}^{0},
$$


trace $=0$, antisymmetric, $\tau_{4}^{(4)}, C=+1$ :

$$
\frac{1}{\sqrt{6}} \sum_{p \in S_{3}} \operatorname{sgn}(p) \mathcal{O}_{\nu_{p(1)} \nu_{p(2)} \nu_{p(3)}}^{0}, \nu_{1}<\nu_{2}<\nu_{3}
$$

trace $=0$, mixed symmetry, $\tau_{2}^{(8)}, C=-1$ :

$$
\frac{1}{2} \mathcal{O}_{\left\langle\left\langle\nu_{1} \nu_{2} \nu_{3}\right\rangle\right\rangle}^{0}, \frac{1}{2 \sqrt{3}} \mathcal{O}_{\left\langle\nu_{1} \nu_{2} \nu_{3}\right\rangle}^{0}, \nu_{1}<\nu_{2}<\nu_{3},
$$

trace $=0$, mixed symmetry, $\tau_{2}^{(8)}, C=+1$ :

$$
\frac{1}{2 \sqrt{3}} \mathcal{O}_{|| \nu_{1} \nu_{2} \nu_{3}||}^{0},-\frac{1}{2} \mathcal{O}_{\left|\nu_{1} \nu_{2} \nu_{3}\right|}^{0}, \nu_{1}<\nu_{2}<\nu_{3},
$$

trace $\neq 0$, mixed symmetry, $\tau_{1}^{(4)}, C=-1$ :

$$
\frac{1}{3 \sqrt{2}} \sum_{\substack{\nu=1 \\ \nu \neq \mu}}^{4} \mathcal{O}_{\langle\langle\mu \nu \nu\rangle\rangle}^{0}, 1 \leq \mu \leq 4
$$

trace $\neq 0$, mixed symmetry, $\tau_{1}^{(4)}, C=+1$ :

$$
\frac{1}{3 \sqrt{6}} \sum_{\substack{\nu=1 \\ \nu \neq \mu}}^{4} \mathcal{O}_{\|\mu \nu \nu\|}^{0}, 1 \leq \mu \leq 4
$$

trace $=0$, mixed symmetry, $\tau_{1}^{(8)}, C=-1$ :

$$
\begin{array}{ll}
\frac{1}{2 \sqrt{3}}\left(\mathcal{O}_{\langle\langle 122\rangle\rangle}^{0}-\mathcal{O}_{\langle\langle 133\rangle\rangle}^{0}\right), & \frac{1}{6}\left(\mathcal{O}_{\langle\langle 122\rangle\rangle}^{0}+\mathcal{O}_{\langle\langle 133\rangle\rangle}^{0}-2 \mathcal{O}_{\langle\langle 144\rangle\rangle}^{0}\right), \\
\frac{1}{2 \sqrt{3}}\left(\mathcal{O}_{\langle\langle 211\rangle\rangle}^{0}-\mathcal{O}_{\langle\langle 233\rangle\rangle}^{0}\right), & \frac{1}{6}\left(\mathcal{O}_{\langle\langle 211\rangle\rangle}^{0}+\mathcal{O}_{\langle\langle 233\rangle\rangle}^{0}-2 \mathcal{O}_{\langle\langle 244\rangle\rangle}^{0}\right), \\
\frac{1}{2 \sqrt{3}}\left(\mathcal{O}_{\langle\langle 311\rangle\rangle}^{0}-\mathcal{O}_{\langle\langle 322\rangle\rangle}^{0}\right), & \frac{1}{6}\left(\mathcal{O}_{\langle\langle 311\rangle\rangle}^{0}+\mathcal{O}_{\langle\langle 322\rangle\rangle}^{0}-2 \mathcal{O}_{\langle\langle 344\rangle\rangle}^{0}\right), \\
\frac{1}{2 \sqrt{3}}\left(\mathcal{O}_{\langle\langle 411\rangle\rangle}^{0}-\mathcal{O}_{\langle\langle 422\rangle\rangle}^{0}\right), & \frac{1}{6}\left(\mathcal{O}_{\langle\langle 411\rangle\rangle}^{0}+\mathcal{O}_{\langle\langle 422\rangle\rangle}^{0}-2 \mathcal{O}_{\langle\langle 433\rangle\rangle}^{0}\right),
\end{array}
$$


trace $=0$, mixed symmetry, $\tau_{1}^{(8)}, C=+1$ :

$$
\begin{aligned}
& \frac{1}{6}\left(\mathcal{O}_{\| 122||}^{0}-\mathcal{O}_{\|133\|}^{0}\right), \frac{1}{6 \sqrt{3}}\left(\mathcal{O}_{\| 122||}^{0}+\mathcal{O}_{\|133\|}^{0}-2 \mathcal{O}_{\| 144 \mid}^{0}\right), \\
& \frac{1}{6}\left(\mathcal{O}_{\| 211||}^{0}-\mathcal{O}_{\|233\|}^{0}\right), \frac{1}{6 \sqrt{3}}\left(\mathcal{O}_{\|211\|}^{0}+\mathcal{O}_{\| 233||}^{0}-2 \mathcal{O}_{\|244\|}^{0}\right) \text {, } \\
& \frac{1}{6}\left(\mathcal{O}_{\| 311||}^{0}-\mathcal{O}_{\|322\|}^{0}\right), \frac{1}{6 \sqrt{3}}\left(\mathcal{O}_{\|311\|}^{0}+\mathcal{O}_{\| 322||}^{0}-2 \mathcal{O}_{\| 344 \mid}^{0}\right), \\
& \frac{1}{6}\left(\mathcal{O}_{\|411\|}^{0}-\mathcal{O}_{\|422\|}^{0}\right), \frac{1}{6 \sqrt{3}}\left(\mathcal{O}_{\|411\|}^{0}+\mathcal{O}_{\| 422||}^{0}-2 \mathcal{O}_{\|433\|}^{0}\right) \text {, }
\end{aligned}
$$

Note that the operators with mixed symmetry and $C=+1$ can be obtained from those with mixed symmetry and $C=-1$ by applying

$$
\frac{1}{\sqrt{3}}(P(\mathrm{id})+2 P((12))) \text {. }
$$

\section{$4.4 n=4$}

For the totally symmetric tensors of rank 4 one can find bases for irreducible subspaces by inspection. For the not totally symmetric rank-4 tensors we first make use of the embedding $H(4) \subset O(4) \subset G L(4)$ and start by decomposing with respect to $G L(4)$. This can be done by standard textbook methods (see, e.g., [6]). For $S_{4}$ we have five Young frames:

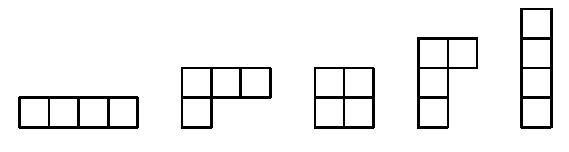

with the first one characterizing the totally symmetric tensors. The corresponding irreducible representations of $G L(4)$ have the dimensions

$$
35 \quad 45 \quad 20 \quad 15 \quad 1
$$

and occur with multiplicity

$$
\begin{array}{lllll}
1 & 3 & 2 & 3 & 1
\end{array}
$$

The subspaces of traceless tensors, which carry the irreducible representation of $O(4)$ corresponding to the particular Young frame, have the dimensions

$$
\begin{array}{lllll}
25 & 30 & 10 & 9 & 1 .
\end{array}
$$


For each of these irreducible representations of $G L(4)$, except for that on the totally symmetric tensors, we construct, following the standard recipes, a projector on an irreducible subspace consisting of tensors which are antisymmetric upon interchange of the second and the fourth index. (Interchange of the second and the fourth index corresponds to charge conjugation, cf. (2.5).) The further decomposition into $H(4)$-irreducible subspaces is then performed by inspection. In this process the knowledge of the above mentioned dimensions proves rather valuable.

In the case of the $G L(4)$ representations which occur with multiplicity greater than one we still need bases for the other irreducible subspaces carrying equivalent $G L(4)$ representations. We get them by acting on the basis already constructed with suitable operators of the form

$$
\sum_{p \in S_{4}} a(p) P(p)
$$

where $P(p)$ was defined in (3.2). The basis obtained in this way has the same transformation properties with respect to $G L(4)$ (and hence also with respect to $H(4))$ as the basis we started with, because the actions of the symmetric group and of the hypercubic group commute. Furthermore the coefficients $a(p)$ will be chosen such that the basis elements are eigenvectors of $(24) \in S_{4}$.

\subsection{1}

We begin by listing $H(4)$-irreducible bases in the space of totally symmetric tensors belonging to the Young frame

trace $\neq 0, \tau_{1}^{(1)}, C=+1:$

$$
\frac{1}{2 \sqrt{2}} \sum_{\mu=1}^{4} \mathcal{O}_{\{\mu \mu \mu \mu\}}^{0}+\frac{1}{\sqrt{2}} \sum_{\substack{\mu, \nu=1 \\ \mu<\nu}}^{4} \mathcal{O}_{\{\mu \mu \nu \nu\}}^{0},
$$

trace $\neq 0, \tau_{1}^{(3)}, C=+1$ :

$$
\begin{aligned}
& \frac{\sqrt{3}}{4}\left(\mathcal{O}_{\{1111\}}^{0}+\mathcal{O}_{\{2222\}}^{0}-\mathcal{O}_{\{3333\}}^{0}-\mathcal{O}_{\{4444\}}^{0}\right)+\frac{\sqrt{3}}{2}\left(\mathcal{O}_{\{1122\}}^{0}-\mathcal{O}_{\{3344\}}^{0}\right) \\
& \frac{\sqrt{3}}{2 \sqrt{2}}\left(\mathcal{O}_{\{3333\}}^{0}-\mathcal{O}_{\{4444\}}^{0}\right)+\frac{\sqrt{3}}{2 \sqrt{2}}\left(\mathcal{O}_{\{1133\}}^{0}-\mathcal{O}_{\{2244\}}^{0}-\mathcal{O}_{\{1144\}}^{0}+\mathcal{O}_{\{2233\}}^{0}\right) \\
& \frac{\sqrt{3}}{2 \sqrt{2}}\left(\mathcal{O}_{\{1111\}}^{0}-\mathcal{O}_{\{2222\}}^{0}\right)+\frac{\sqrt{3}}{2 \sqrt{2}}\left(\mathcal{O}_{\{1133\}}^{0}-\mathcal{O}_{\{2244\}}^{0}+\mathcal{O}_{\{1144\}}^{0}-\mathcal{O}_{\{2233\}}^{0}\right)
\end{aligned}
$$


trace $\neq 0, \tau_{3}^{(6)}, C=+1$ :

$$
\frac{\sqrt{3}}{\sqrt{2}}\left(\mathcal{O}_{\{\mu \mu \mu \nu\}}^{0}+\mathcal{O}_{\{\nu \nu \nu \mu\}}^{0}+\mathcal{O}_{\left\{\nu_{1} \nu_{1} \mu \nu\right\}}^{0}+\mathcal{O}_{\left\{\nu_{2} \nu_{2} \mu \nu\right\}}^{0}\right), 1 \leq \mu<\nu \leq 4
$$

where $\nu_{1}, \nu_{2}$ are such that $\nu_{1}<\nu_{2}$ and $\left\{\mu, \nu, \nu_{1}, \nu_{2}\right\}=\{1,2,3,4\}$,

$$
\begin{aligned}
\text { trace }=0, \tau_{1}^{(1)}, C=+1: & \\
& \frac{1}{2 \sqrt{2}} \sum_{\mu=1}^{4} \mathcal{O}_{\{\mu \mu \mu \mu\}}^{0}-\frac{1}{\sqrt{2}} \sum_{\substack{\mu, \nu=1 \\
\mu<\nu}}^{4} \mathcal{O}_{\{\mu \mu \nu \nu\}}^{0},
\end{aligned}
$$

trace $=0, \tau_{1}^{(3)}, C=+1$ :

$$
\begin{gathered}
\frac{1}{4}\left(\mathcal{O}_{\{1111\}}^{0}+\mathcal{O}_{\{2222\}}^{0}-\mathcal{O}_{\{3333\}}^{0}-\mathcal{O}_{\{4444\}}^{0}\right)-\frac{3}{2}\left(\mathcal{O}_{\{1122\}}^{0}-\mathcal{O}_{\{3344\}}^{0}\right) \\
\frac{1}{2 \sqrt{2}}\left(\mathcal{O}_{\{3333\}}^{0}-\mathcal{O}_{\{4444\}}^{0}\right)-\frac{3}{2 \sqrt{2}}\left(\mathcal{O}_{\{1133\}}^{0}-\mathcal{O}_{\{2244\}}^{0}-\mathcal{O}_{\{1144\}}^{0}+\mathcal{O}_{\{2233\}}^{0}\right) \\
\frac{1}{2 \sqrt{2}}\left(\mathcal{O}_{\{1111\}}^{0}-\mathcal{O}_{\{2222\}}^{0}\right)-\frac{3}{2 \sqrt{2}}\left(\mathcal{O}_{\{1133\}}^{0}-\mathcal{O}_{\{2244\}}^{0}+\mathcal{O}_{\{1144\}}^{0}-\mathcal{O}_{\{2233\}}^{0}\right)
\end{gathered}
$$

trace $=0, \tau_{3}^{(6)}, C=+1$ :

$$
\frac{1}{\sqrt{2}}\left(\mathcal{O}_{\{\mu \mu \mu \nu\}}^{0}+\mathcal{O}_{\{\nu \nu \nu \mu\}}^{0}\right)-\frac{3}{\sqrt{2}}\left(\mathcal{O}_{\left\{\nu_{1} \nu_{1} \mu \nu\right\}}^{0}+\mathcal{O}_{\left\{\nu_{2} \nu_{2} \mu \nu\right\}}^{0}\right), 1 \leq \mu<\nu \leq 4,
$$

where $\nu_{1}, \nu_{2}$ are such that $\nu_{1}<\nu_{2}$ and $\left\{\mu, \nu, \nu_{1}, \nu_{2}\right\}=\{1,2,3,4\}$,

$$
\begin{gathered}
\text { trace }=0, \tau_{1}^{(2)}, C=+1: \\
\frac{\sqrt{3}}{\sqrt{2}}\left(\mathcal{O}_{\{1122\}}^{0}+\mathcal{O}_{\{3344\}}^{0}-\mathcal{O}_{\{1133\}}^{0}-\mathcal{O}_{\{2244\}}^{0}\right) \\
-\frac{1}{\sqrt{2}}\left(\mathcal{O}_{\{1122\}}^{0}+\mathcal{O}_{\{3344\}}^{0}+\mathcal{O}_{\{1133\}}^{0}+\mathcal{O}_{\{2244\}}^{0}-2 \mathcal{O}_{\{1144\}}^{0}-2 \mathcal{O}_{\{2233\}}^{0}\right),
\end{gathered}
$$

trace $=0, \tau_{2}^{(1)}, C=+1$ :

$$
2 \sqrt{6} \mathcal{O}_{\{1234\}}^{0}
$$

trace $=0, \tau_{1}^{(6)}, C=+1$ :

$$
\sqrt{2}\left(\mathcal{O}_{\{\mu \mu \mu \nu\}}^{0}-\mathcal{O}_{\{\nu \nu \nu \mu\}}^{0}\right), 1 \leq \mu<\nu \leq 4
$$


trace $=0, \tau_{2}^{(6)}, C=+1:$

$$
\sqrt{6}\left(\mathcal{O}_{\left\{\nu_{1} \nu_{1} \mu \nu\right\}}^{0}-\mathcal{O}_{\left\{\nu_{2} \nu_{2} \mu \nu\right\}}^{0}\right), 1 \leq \mu<\nu \leq 4
$$

where $\nu_{1}, \nu_{2}$ are such that $\nu_{1}<\nu_{2}$ and $\left\{\mu, \nu, \nu_{1}, \nu_{2}\right\}=\{1,2,3,4\}$.

\section{4 .2}

In order to describe a basis for an irreducible subspace (with respect to $G L(4)$ ) corresponding to $\square \square$ we define

$$
\mathcal{O}_{\lambda \rho}^{\mu \nu}=N_{\lambda \rho}\left(\mathcal{O}_{\lambda \mu \rho \nu}^{0}-\mathcal{O}_{\lambda \nu \rho \mu}^{0}+\mathcal{O}_{\rho \mu \lambda \nu}^{0}-\mathcal{O}_{\rho \nu \lambda \mu}^{0}\right)
$$

with

$$
N_{\lambda \rho}= \begin{cases}1 / 2 & \text { for } \lambda \neq \rho \\ 1 / \sqrt{8} & \text { for } \quad \lambda=\rho\end{cases}
$$

With respect to $H(4)$ one gets the following irreducible subspaces of this $G L(4)$ irreducible space.

trace $\neq 0, \tau_{1}^{(3)}, C=-1$ :

$$
\begin{gathered}
\frac{1}{\sqrt{3}}\left(\mathcal{O}_{12}^{12}-\mathcal{O}_{23}^{23}-\mathcal{O}_{24}^{24}\right) \\
\frac{1}{2 \sqrt{6}}\left(-\mathcal{O}_{12}^{12}-3 \mathcal{O}_{13}^{13}-2 \mathcal{O}_{23}^{23}+\mathcal{O}_{24}^{24}+3 \mathcal{O}_{34}^{34}\right) \\
\frac{1}{2 \sqrt{2}}\left(\mathcal{O}_{12}^{12}+\mathcal{O}_{13}^{13}+2 \mathcal{O}_{14}^{14}+\mathcal{O}_{24}^{24}+\mathcal{O}_{34}^{34}\right)
\end{gathered}
$$

trace $\neq 0, \tau_{1}^{(6)}, C=-1$ :

$$
-\frac{1}{\sqrt{3}}\left(\mathcal{O}_{\nu \nu}^{\nu \lambda}+\mathcal{O}_{\lambda \lambda}^{\nu \lambda}\right)+\frac{1}{2 \sqrt{6}} \sum_{i=1}^{2}\left(\mathcal{O}_{\mu_{i} \lambda}^{\mu_{i} \nu}-\mathcal{O}_{\mu_{i} \nu}^{\mu_{i} \lambda}-\sqrt{2} \mathcal{O}_{\mu_{i} \mu_{i}}^{\nu \lambda}\right), \nu<\lambda
$$

trace $\neq 0, \tau_{3}^{(6)}, C=-1$ :

$$
\frac{1}{2}\left(\mathcal{O}_{\nu \nu}^{\nu \lambda}-\mathcal{O}_{\lambda \lambda}^{\nu \lambda}\right)+\frac{1}{2 \sqrt{2}} \sum_{i=1}^{2}\left(\mathcal{O}_{\mu_{i} \lambda}^{\mu_{i} \nu}+\mathcal{O}_{\mu_{i} \nu}^{\mu_{i} \lambda}\right), \nu<\lambda
$$


trace $=0, \tau_{2}^{(3)}, C=-1:$

$$
\begin{gathered}
\frac{1}{\sqrt{3}}\left(\mathcal{O}_{23}^{14}+\mathcal{O}_{13}^{24}+\mathcal{O}_{12}^{34}\right) \\
\frac{1}{2 \sqrt{6}}\left(3 \mathcal{O}_{24}^{13}+\mathcal{O}_{23}^{14}+3 \mathcal{O}_{14}^{23}+\mathcal{O}_{13}^{24}-2 \mathcal{O}_{12}^{34}\right) \\
\frac{1}{2 \sqrt{2}}\left(2 \mathcal{O}_{34}^{12}+\mathcal{O}_{24}^{13}+\mathcal{O}_{23}^{14}-\mathcal{O}_{14}^{23}-\mathcal{O}_{13}^{24}\right)
\end{gathered}
$$

trace $=0, \tau_{3}^{(3)}, C=-1$ :

$$
\begin{gathered}
\frac{1}{\sqrt{3}}\left(\mathcal{O}_{12}^{12}-\mathcal{O}_{13}^{13}+\mathcal{O}_{23}^{23}\right) \\
\frac{1}{2 \sqrt{6}}\left(-2 \mathcal{O}_{12}^{12}-\mathcal{O}_{13}^{13}+3 \mathcal{O}_{14}^{14}+\mathcal{O}_{23}^{23}-3 \mathcal{O}_{24}^{24}\right) \\
\frac{1}{2 \sqrt{2}}\left(-\mathcal{O}_{13}^{13}+\mathcal{O}_{14}^{14}-\mathcal{O}_{23}^{23}+\mathcal{O}_{24}^{24}-2 \mathcal{O}_{34}^{34}\right)
\end{gathered}
$$

trace $=0, \tau_{1}^{(6)}, C=-1:$

$$
\frac{1}{\sqrt{6}}\left(\mathcal{O}_{\nu \nu}^{\nu \lambda}+\mathcal{O}_{\lambda \lambda}^{\nu \lambda}\right)+\frac{1}{2 \sqrt{3}} \sum_{i=1}^{2}\left(\mathcal{O}_{\mu_{i} \lambda}^{\mu_{i} \nu}-\mathcal{O}_{\mu_{i} \nu}^{\mu_{i} \lambda}-\sqrt{2} \mathcal{O}_{\mu_{i} \mu_{i}}^{\nu \lambda}\right), \nu<\lambda
$$

trace $=0, \tau_{2}^{(6)}, C=-1$ :

$$
\frac{1}{2} \sum_{i=1}^{2}(-1)^{i+1}\left(\mathcal{O}_{\mu_{i} \lambda}^{\mu_{i} \nu}+\mathcal{O}_{\mu_{i} \nu}^{\mu_{i} \lambda}\right), \nu<\lambda
$$

trace $=0, \tau_{3}^{(6)}, C=-1:$

$$
\frac{1}{2}\left(\mathcal{O}_{\nu \nu}^{\nu \lambda}-\mathcal{O}_{\lambda \lambda}^{\nu \lambda}\right)-\frac{1}{2 \sqrt{2}} \sum_{i=1}^{2}\left(\mathcal{O}_{\mu_{i} \lambda}^{\mu_{i} \nu}+\mathcal{O}_{\mu_{i} \nu}^{\mu_{i} \lambda}\right), \nu<\lambda
$$

trace $=0, \tau_{4}^{(6)}, C=-1:$

$$
\frac{1}{2} \sum_{i=1}^{2}(-1)^{i+1}\left(\mathcal{O}_{\mu_{i} \lambda}^{\mu_{i} \nu}-\mathcal{O}_{\mu_{i} \nu}^{\mu_{i} \lambda}-\sqrt{2} \mathcal{O}_{\mu_{i} \mu_{i}}^{\nu \lambda}\right), \nu<\lambda
$$

For $\nu<\lambda$ the indices $\mu_{1}, \mu_{2}$ are such that $\mu_{1}<\mu_{2}$ and $\left\{\nu, \lambda, \mu_{1}, \mu_{2}\right\}=\{1,2,3,4\}$. 
Applying the operators

$$
\begin{gathered}
\frac{1}{\sqrt{3}}(P(\mathrm{id})-2 P((12))), \\
\frac{1}{\sqrt{6}}(P(\mathrm{id})+P((12))-3 P((23))),
\end{gathered}
$$

respectively, to the basis vectors (4.35) - (4.43) one obtains an orthonormal system of vectors which transform under $H(4)$ exactly like (4.35) - (4.43) but have $C=+1$.

\section{4 .3}

In order to describe a basis for an irreducible subspace (with respect to $G L(4)$ ) corresponding to this Young frame we define

$$
\begin{aligned}
\tilde{\mathcal{O}}_{i j}=N_{i j}( & \mathcal{O}_{\mu_{i} \mu_{j} \nu_{i} \nu_{j}}^{0}-\mathcal{O}_{\nu_{i} \mu_{j} \mu_{i} \nu_{j}}^{0}-\mathcal{O}_{\mu_{i} \nu_{j} \nu_{i} \mu_{j}}^{0}+\mathcal{O}_{\nu_{i} \nu_{j} \mu_{i} \mu_{j}}^{0} \\
& \left.+\mathcal{O}_{\mu_{j} \mu_{i} \nu_{j} \nu_{i}}^{0}-\mathcal{O}_{\nu_{j} \mu_{i} \mu_{j} \nu_{i}}^{0}-\mathcal{O}_{\mu_{j} \nu_{i} \nu_{j} \mu_{i}}^{0}+\mathcal{O}_{\nu_{j} \nu_{i} \mu_{j} \mu_{i}}^{0}\right)
\end{aligned}
$$

with

$$
N_{i j}= \begin{cases}1 / \sqrt{8} & \text { for } i \neq j \\ 1 / 4 & \text { for } i=j\end{cases}
$$

The indices $i, j \in\{1,2,3,4,5,6\}$ enumerate pairs $\mu \nu$ with $\mu<\nu$ according to the scheme

$$
\begin{array}{ccccccc}
i & 1 & 2 & 3 & 4 & 5 & 6 \\
\mu_{i} & 1 & 1 & 1 & 2 & 2 & 3 \\
\nu_{i} & 2 & 3 & 4 & 3 & 4 & 4
\end{array}
$$

Note that $\tilde{\mathcal{O}}_{i j}=\tilde{\mathcal{O}}_{j i}$.

With respect to $H(4)$ one gets the following irreducible subspaces of the $G L(4)$ irreducible space.

trace $\neq 0, \tau_{1}^{(1)}, C=-1:$

$$
\frac{1}{\sqrt{6}}\left(\tilde{\mathcal{O}}_{11}+\cdots+\tilde{\mathcal{O}}_{66}\right)
$$


trace $\neq 0, \tau_{1}^{(3)}, C=-1:$

$$
\frac{1}{\sqrt{2}}\left(\tilde{\mathcal{O}}_{11}-\tilde{\mathcal{O}}_{66}\right), \frac{1}{\sqrt{2}}\left(\tilde{\mathcal{O}}_{22}-\tilde{\mathcal{O}}_{55}\right), \frac{1}{\sqrt{2}}\left(\tilde{\mathcal{O}}_{33}-\tilde{\mathcal{O}}_{44}\right)
$$

trace $\neq 0, \tau_{3}^{(6)}, C=-1$ :

$$
\begin{array}{ll}
\frac{1}{\sqrt{2}}\left(\tilde{\mathcal{O}}_{12}+\tilde{\mathcal{O}}_{56}\right) \quad, \quad \frac{1}{\sqrt{2}}\left(\tilde{\mathcal{O}}_{13}-\tilde{\mathcal{O}}_{46}\right), \frac{1}{\sqrt{2}}\left(\tilde{\mathcal{O}}_{14}-\tilde{\mathcal{O}}_{36}\right) \\
\frac{1}{\sqrt{2}}\left(\tilde{\mathcal{O}}_{15}+\tilde{\mathcal{O}}_{26}\right), \frac{1}{\sqrt{2}}\left(\tilde{\mathcal{O}}_{23}+\tilde{\mathcal{O}}_{45}\right), \frac{1}{\sqrt{2}}\left(\tilde{\mathcal{O}}_{24}+\tilde{\mathcal{O}}_{35}\right)
\end{array}
$$

trace $=0, \tau_{1}^{(2)}, C=-1$ :

$$
\frac{1}{\sqrt{12}}\left(-2 \tilde{\mathcal{O}}_{11}-2 \tilde{\mathcal{O}}_{66}+\tilde{\mathcal{O}}_{22}+\tilde{\mathcal{O}}_{33}+\tilde{\mathcal{O}}_{44}+\tilde{\mathcal{O}}_{55}\right), \frac{1}{2}\left(\tilde{\mathcal{O}}_{33}+\tilde{\mathcal{O}}_{44}-\tilde{\mathcal{O}}_{22}-\tilde{\mathcal{O}}_{55}\right)
$$

trace $=0, \tau_{2}^{(2)}, C=-1$ :

$$
\frac{1}{\sqrt{2}}\left(\tilde{\mathcal{O}}_{16}+\tilde{\mathcal{O}}_{25}\right), \frac{1}{\sqrt{6}}\left(\tilde{\mathcal{O}}_{16}-\tilde{\mathcal{O}}_{25}-2 \tilde{\mathcal{O}}_{34}\right)
$$

trace $=0, \tau_{2}^{(6)}, C=-1$ :

$$
\begin{array}{ll}
\frac{1}{\sqrt{2}}\left(\tilde{\mathcal{O}}_{12}-\tilde{\mathcal{O}}_{56}\right) \quad, \quad \frac{1}{\sqrt{2}}\left(\tilde{\mathcal{O}}_{13}+\tilde{\mathcal{O}}_{46}\right), \frac{1}{\sqrt{2}}\left(\tilde{\mathcal{O}}_{14}+\tilde{\mathcal{O}}_{36}\right) \\
\frac{1}{\sqrt{2}}\left(\tilde{\mathcal{O}}_{15}-\tilde{\mathcal{O}}_{26}\right) \quad, \quad \frac{1}{\sqrt{2}}\left(\tilde{\mathcal{O}}_{23}-\tilde{\mathcal{O}}_{45}\right), \frac{1}{\sqrt{2}}\left(\tilde{\mathcal{O}}_{24}-\tilde{\mathcal{O}}_{35}\right)
\end{array}
$$

Applying the operator

$$
\frac{1}{\sqrt{3}}(P(\mathrm{id})-2 P((12)))
$$

to the basis vectors $(4.48)-(4.53)$ one obtains an orthonormal system of vectors which transform under $H(4)$ exactly like (4.48) - (4.53) but have $C=+1$. 


\subsection{4}

In order to describe a basis for an irreducible subspace (with respect to $G L(4)$ ) corresponding to this Young frame we define

$$
\hat{\mathcal{O}}_{\mu \nu}=\frac{1}{\sqrt{6}} \sum_{p \in S_{3}} \operatorname{sgn}(p) \mathcal{O}_{\nu_{p(1)} \nu_{p(2)} \mu \nu_{p(3)}}^{0} .
$$

The indices $\nu_{1}, \nu_{2}, \nu_{3}$ are determined by $\nu$ through the conditions $\nu_{1}<\nu_{2}<\nu_{3}$ and $\left\{\nu_{1}, \nu_{2}, \nu_{3}, \nu\right\}=\{1,2,3,4\}$.

With respect to $H(4)$ one gets the following irreducible subspaces of the $G L(4)$ irreducible space.

trace $\neq 0, \tau_{1}^{(6)}, C=-1$ :

$$
\begin{array}{lll}
\frac{1}{\sqrt{2}}\left(\hat{\mathcal{O}}_{12}+\hat{\mathcal{O}}_{21}\right), & \frac{1}{\sqrt{2}}\left(\hat{\mathcal{O}}_{13}-\hat{\mathcal{O}}_{31}\right), \frac{1}{\sqrt{2}}\left(\hat{\mathcal{O}}_{14}+\hat{\mathcal{O}}_{41}\right) \\
\frac{1}{\sqrt{2}}\left(\hat{\mathcal{O}}_{23}+\hat{\mathcal{O}}_{32}\right), & \frac{1}{\sqrt{2}}\left(\hat{\mathcal{O}}_{24}-\hat{\mathcal{O}}_{42}\right), & \frac{1}{\sqrt{2}}\left(\hat{\mathcal{O}}_{34}+\hat{\mathcal{O}}_{43}\right)
\end{array}
$$

trace $=0, \tau_{4}^{(3)}, C=-1:$

$$
\frac{1}{2}\left(\hat{\mathcal{O}}_{11}-\hat{\mathcal{O}}_{22}-\hat{\mathcal{O}}_{33}+\hat{\mathcal{O}}_{44}\right), \frac{1}{\sqrt{2}}\left(\hat{\mathcal{O}}_{33}+\hat{\mathcal{O}}_{44}\right), \frac{1}{\sqrt{2}}\left(\hat{\mathcal{O}}_{11}+\hat{\mathcal{O}}_{22}\right)
$$

trace $=0, \tau_{4}^{(6)}, C=-1$ :

$$
\begin{array}{lll}
\frac{1}{\sqrt{2}}\left(\hat{\mathcal{O}}_{12}-\hat{\mathcal{O}}_{21}\right), & \frac{1}{\sqrt{2}}\left(\hat{\mathcal{O}}_{13}+\hat{\mathcal{O}}_{31}\right), \frac{1}{\sqrt{2}}\left(\hat{\mathcal{O}}_{14}-\hat{\mathcal{O}}_{41}\right) \\
\frac{1}{\sqrt{2}}\left(\hat{\mathcal{O}}_{23}-\hat{\mathcal{O}}_{32}\right), & \frac{1}{\sqrt{2}}\left(\hat{\mathcal{O}}_{24}+\hat{\mathcal{O}}_{42}\right), & \frac{1}{\sqrt{2}}\left(\hat{\mathcal{O}}_{34}-\hat{\mathcal{O}}_{43}\right)
\end{array}
$$

Applying the operators

$$
\begin{gathered}
\frac{1}{2 \sqrt{2}}(2 P(\mathrm{id})-3 P((23))-3 P((34))), \\
\sqrt{\frac{3}{8}}(P((23))-P((34))),
\end{gathered}
$$


respectively, to the basis vectors $(4.56)$ - (4.58) one obtains two orthonormal systems of vectors which transform under $H(4)$ exactly like (4.56) - (4.58). Using (4.59) one gets $C=-1$, whereas (4.60) leads to $C=+1$.

\subsection{5}

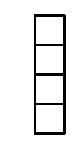

The irreducible subspace corresponding to this Young frame is one-dimensional and carries the following representation of $H(4)$.

$$
\begin{aligned}
& \text { trace }=0, \tau_{4}^{(1)}, C=-1: \\
& \quad \frac{1}{\sqrt{24}} \sum_{p \in S_{4}} \operatorname{sgn}(p) \mathcal{O}_{p(1) \cdots p(4)}^{0} .
\end{aligned}
$$

\section{$5 \quad$ Axial Operators}

The transformation law 2.19) of the operators $\mathcal{O}_{\mu_{1} \mu_{2} \ldots \mu_{n}}^{5}$ differs from the transformation law (2.17) only by the additional factor of $\operatorname{det}(T(a, \pi))$. Since the mapping

$$
(a, \pi) \rightarrow \operatorname{det}(T(a, \pi))
$$

is the (one-dimensional) irreducible representation $\tau_{4}^{(1)}$ bases of irreducible subspaces can be constructed from the operators $\mathcal{O}_{\mu_{1} \mu_{2} \ldots \mu_{n}}^{5}$ in exactly the same manner as from the operators $\mathcal{O}_{\mu_{1} \mu_{2} \ldots \mu_{n}}^{0}$. The only difference is that $C$-parity changes sign and the 
representations acting in these subspaces have to be multiplied by $\tau_{4}^{(1)}$. One finds

$$
\begin{gathered}
\tau_{1}^{(1)} \rightarrow \tau_{4}^{(1)} \otimes \tau_{1}^{(1)}=\tau_{4}^{(1)}, \quad \tau_{2}^{(1)} \rightarrow \tau_{4}^{(1)} \otimes \tau_{2}^{(1)}=\tau_{3}^{(1)}, \\
\tau_{3}^{(1)} \rightarrow \tau_{4}^{(1)} \otimes \tau_{3}^{(1)}=\tau_{2}^{(1)}, \quad \tau_{4}^{(1)} \rightarrow \tau_{4}^{(1)} \otimes \tau_{4}^{(1)}=\tau_{1}^{(1)}, \\
\tau_{1}^{(2)} \rightarrow \tau_{4}^{(1)} \otimes \tau_{1}^{(2)}=\tau_{2}^{(2)}, \quad \tau_{2}^{(2)} \rightarrow \tau_{4}^{(1)} \otimes \tau_{2}^{(2)}=\tau_{1}^{(2)}, \\
\tau_{1}^{(3)} \rightarrow \tau_{4}^{(1)} \otimes \tau_{1}^{(3)}=\tau_{4}^{(3)}, \quad \tau_{2}^{(3)} \rightarrow \tau_{4}^{(1)} \otimes \tau_{2}^{(3)}=\tau_{3}^{(3)}, \\
\tau_{3}^{(3)} \rightarrow \tau_{4}^{(1)} \otimes \tau_{3}^{(3)}=\tau_{2}^{(3)}, \quad \tau_{4}^{(3)} \rightarrow \tau_{4}^{(1)} \otimes \tau_{4}^{(3)}=\tau_{1}^{(3)}, \\
\tau_{1}^{(4)} \rightarrow \tau_{4}^{(1)} \otimes \tau_{1}^{(4)}=\tau_{4}^{(4)}, \quad \tau_{2}^{(4)} \rightarrow \tau_{4}^{(1)} \otimes \tau_{2}^{(4)}=\tau_{3}^{(4)}, \\
\tau_{3}^{(4)} \rightarrow \tau_{4}^{(1)} \otimes \tau_{3}^{(4)}=\tau_{2}^{(4)}, \quad \tau_{4}^{(4)} \rightarrow \tau_{4}^{(1)} \otimes \tau_{4}^{(4)}=\tau_{1}^{(4)}, \\
\tau_{1}^{(6)} \rightarrow \tau_{4}^{(1)} \otimes \tau_{1}^{(6)}=\tau_{1}^{(6)}, \quad \tau_{2}^{(6)} \rightarrow \tau_{4}^{(1)} \otimes \tau_{2}^{(6)}=\tau_{2}^{(6)}, \\
\tau_{3}^{(6)} \rightarrow \tau_{4}^{(1)} \otimes \tau_{3}^{(6)}=\tau_{4}^{(6)}, \quad \tau_{4}^{(6)} \rightarrow \tau_{4}^{(1)} \otimes \tau_{4}^{(6)}=\tau_{3}^{(6)}, \\
\tau_{1}^{(8)} \rightarrow \tau_{4}^{(1)} \otimes \tau_{1}^{(8)}=\tau_{2}^{(8)}, \quad \tau_{2}^{(8)} \rightarrow \tau_{4}^{(1)} \otimes \tau_{2}^{(8)}=\tau_{1}^{(8)} .
\end{gathered}
$$

\section{$6 \quad$ Mixing}

As one sees, quite a number of pairs $\left(\tau_{k}^{(l)}, C\right)$ occur more than once leading to potential mixing problems. In order to find out which operators could mix one needs bases for the equivalent subspaces which transform identically under $H(4)$. The bases given above do not always have this property, but constructing appropriate basis transformations should present no difficulties in specific cases of interest. In this connection it is useful to remember that $H(4)$ is generated by the group elements (2.11) - (2.13) so that the transformation behaviour of a given basis has to be checked only for these three elements. We shall quote only a few examples relevant for our own numerical work [4], where we have studied (among others) the following operators and representations.

trace $=0$, symmetric, $\tau_{1}^{(8)}, C=-1$ :

$$
\begin{aligned}
& \mathcal{O}_{\{411\}}^{0}-\frac{1}{2}\left(\mathcal{O}_{\{422\}}^{0}+\mathcal{O}_{\{433\}}^{0}\right) \\
& \quad=\frac{\sqrt{3}}{2 \sqrt{2}} \cdot \frac{\sqrt{3}}{\sqrt{2}}\left(\mathcal{O}_{\{411\}}^{0}-\mathcal{O}_{\{422\}}^{0}\right)+\frac{1}{2 \sqrt{2}} \cdot \frac{1}{\sqrt{2}}\left(\mathcal{O}_{\{411\}}^{0}+\mathcal{O}_{\{422\}}^{0}-2 \mathcal{O}_{\{433\}}^{0}\right) .
\end{aligned}
$$


Operators transforming identically are

$$
\frac{1}{3 \sqrt{2}}\left(\mathcal{O}_{\langle\langle 411\rangle\rangle}^{0}-\frac{1}{2}\left(\mathcal{O}_{\langle\langle 422\rangle\rangle}^{0}+\mathcal{O}_{\langle\langle 433\rangle\rangle}^{0}\right)\right)
$$

and

$$
-\frac{1}{4 \sqrt{6}}\left(\mathcal{O}_{|| 123||}^{5}+3 \mathcal{O}_{|123|}^{5}\right) .
$$

trace $=0$, symmetric, $\tau_{1}^{(2)}, C=+1$ :

$$
\begin{aligned}
& \mathcal{O}_{\{1144\}}^{0}+\mathcal{O}_{\{2233\}}^{0}-\mathcal{O}_{\{1133\}}^{0}-\mathcal{O}_{\{2244\}}^{0} \\
& =\frac{1}{\sqrt{6}} \cdot \frac{\sqrt{3}}{\sqrt{2}}\left(\mathcal{O}_{\{1122\}}^{0}+\mathcal{O}_{\{3344\}}^{0}-\mathcal{O}_{\{1133\}}^{0}-\mathcal{O}_{\{2244\}}^{0}\right) \\
& \quad-\frac{1}{\sqrt{2}} \cdot \frac{1}{\sqrt{2}}\left(\mathcal{O}_{\{1122\}}^{0}+\mathcal{O}_{\{3344\}}^{0}+\mathcal{O}_{\{1133\}}^{0}+\mathcal{O}_{\{2244\}}^{0}-2 \mathcal{O}_{\{1144\}}^{0}-2 \mathcal{O}_{\{2233\}}^{0}\right) .
\end{aligned}
$$

Operators transforming identically are

$$
\begin{aligned}
\frac{1}{6 \sqrt{2}}( & -\mathcal{O}_{1144}^{0}-\mathcal{O}_{4114}^{0}-\mathcal{O}_{1441}^{0}-\mathcal{O}_{4411}^{0}+2 \mathcal{O}_{1414}^{0}+2 \mathcal{O}_{4141}^{0} \\
& -\mathcal{O}_{2233}^{0}-\mathcal{O}_{3223}^{0}-\mathcal{O}_{2332}^{0}-\mathcal{O}_{3322}^{0}+2 \mathcal{O}_{2323}^{0}+2 \mathcal{O}_{3232}^{0} \\
& +\mathcal{O}_{1133}^{0}+\mathcal{O}_{3113}^{0}+\mathcal{O}_{1331}^{0}+\mathcal{O}_{3311}^{0}-2 \mathcal{O}_{1313}^{0}-2 \mathcal{O}_{3131}^{0} \\
& \left.+\mathcal{O}_{2244}^{0}+\mathcal{O}_{4224}^{0}+\mathcal{O}_{2442}^{0}+\mathcal{O}_{4422}^{0}-2 \mathcal{O}_{2424}^{0}-2 \mathcal{O}_{4242}^{0}\right)
\end{aligned}
$$

and

$$
\begin{aligned}
& \frac{1}{2 \sqrt{6}}\left(\mathcal{O}_{1234}^{5}-\mathcal{O}_{3214}^{5}-\mathcal{O}_{1432}^{5}+\mathcal{O}_{3412}^{5}+\mathcal{O}_{2143}^{5}-\mathcal{O}_{4123}^{5}-\mathcal{O}_{2341}^{5}+\mathcal{O}_{4321}^{5}\right. \\
& \left.\quad+\mathcal{O}_{1243}^{5}-\mathcal{O}_{4213}^{5}-\mathcal{O}_{1342}^{5}+\mathcal{O}_{4312}^{5}+\mathcal{O}_{2134}^{5}-\mathcal{O}_{3124}^{5}-\mathcal{O}_{2431}^{5}+\mathcal{O}_{3421}^{5}\right)
\end{aligned}
$$

trace $=0$, mixed symmetry, $\tau_{1}^{(8)}, C=+1$ :

$$
2 \mathcal{O}_{2\{14\}}^{5}-\mathcal{O}_{1\{24\}}^{5}-\mathcal{O}_{4\{12\}}^{5}=-\frac{1}{2} \mathcal{O}_{\langle 124\rangle}^{5} .
$$

An operator transforming identically is

$$
\frac{\sqrt{3}}{6}\left(\mathcal{O}_{\| 311||}^{0}-\mathcal{O}_{|| 344||}^{0}\right) .
$$




\section{Discussion}

The results presented in this paper enable us to determine the mixings allowed by symmetry among the lattice operators that are used to calculate hadronic structure functions. Mixing with lower-dimensional operators leads to contributions which diverge like a power of the inverse lattice spacing in the continuum limit. These have either to be subtracted nonperturbatively (a difficult task) or to be avoided by a suitable choice of the operators. Mixing with operators of the same dimension should be tractable by perturbation theory although a nonperturbative treatment seems favourable. In any case, it leads to additional uncertainties, but as $n$ grows, operators with no mixing at all require more and more nonvanishing momentum components in the calculation of their forward hadronic matrix elements, which makes their Monte Carlo evaluation increasingly difficult. So some kind of compromise is needed.

For the cases listed in section 6 a 1-loop calculation in lattice perturbation theory leads to mixing only between the operators (6.1) and (6.2). Moreover, the corresponding mixing coefficient turns out to be rather small at the presently used values of the coupling constant [3, 4]. Hence mixing with operators of the same dimension seems to be relatively harmless. However, the nasty mixing with lower-dimensional operators will become unavoidable when dealing with operators of higher spin.

\section{Acknowledgements}

This work is supported by the Deutsche Forschungsgemeinschaft and the European Community under contract number CHRX-CT92-0051.

\section{References}

[1] G. Martinelli and C.T. Sachrajda, Nucl. Phys. B306 (1988) 865; Nucl. Phys. B316 (1989) 355

[2] S. Capitani and G. Rossi, Nucl. Phys. B433 (1995) 351

[3] G. Beccarini, M. Bianchi, S. Capitani and G. Rossi, Nucl. Phys. B456 (1995) 271 
[4] M. Göckeler, R. Horsley, E.-M. Ilgenfritz, H. Perlt, P. Rakow, G. Schierholz and A. Schiller, Preprint DESY 95-128, HLRZ 95-36, HUB-EP-95/9 (hep-lat/9508004), to appear in Phys. Rev. D

[5] M. Baake, B. Gemünden and R. Oedingen, J. Math. Phys. 23 (1982) 944

[6] W. Miller, Symmetry Groups and Their Applications (Academic Press, New York and London, 1972)

[7] J.E. Mandula, G. Zweig and J. Govaerts, Nucl. Phys. B228 (1983) 91 